\title{
Carotenoid Profiles of Dried Herbs, Water Infusions and Alcoholic Tinctures of Calendula Flower and Catnip, Dandelion, Stinging Nettle, and Violet Leaves
} Kiersten Olsen ${ }^{1}$, Sara Arscott ${ }^{2}$ and Sherry A Tanumihardjo ${ }^{1^{*}}$

${ }^{1}$ Interdepartmental Graduate Program in Nutritional Sciences, Department of Nutritional Sciences, University of Wisconsin-Madison 1415 Linden Drive, Madison, WI 537061571, USA

${ }^{2}$ Standard Process, Inc., Palmyra, WI 53156, USA

\begin{abstract}
Herbs have been used for centuries to help with various ailments in cultures throughout the world. Herbal water infusions and alcoholic tinctures are two processes that are still used today. Five herbs, Calendula flower (Calendula officinalis L.) and Catnip (Nepeta cataria), Dandelion (Taraxacum officinale F. Weber ex Wiggers), Stinging Nettle (Urtica dioica L.) and Violet leaves (Viola odorata), were analyzed for carotenoid content in three forms: raw-dried herb, water infusion, and alcoholic tincture. Carotenoids infer putative health benefits and act as potential antioxidants and vitamin A precursors. Carotenoid content analysis of herbal preparations adds to current knowledge of which forms deliver the greatest amounts. In order to evaluate carotenoid content, high pressure liquid chromatographic analyses were performed. The concentrations of all-trans- $\beta$-carotene, 9 - and 13 -cis- $\beta$-carotene, zeaxanthin, and lutein within each herbal form were determined. As expected among the preparations evaluated, the raw-dried herb showed the highest mean concentrations of all five carotenoids. The mean carotenoid concentrations in the herbal tincture and infusion forms did not always reflect the same relative profile as the dried herb.
\end{abstract}

Keywords: Alcoholic tinctures; Carotenoids; Marc; Menstruum; Water infusions

Abbreviations: CM: Commercially Available; HPLC: High Pressure Liquid Chromatography; HG: Homegrown; VA: Vitamin A

\section{Introduction}

Vitamin A (VA) is an essential nutrient with a number of healthpromoting benefits. VA is a fat-soluble compound that is involved in the regulation and promotion of growth and differentiation of many cells especially in the eyes and lungs [1]. VA can be formed from specific carotenoids, which are commonly called provitamin A carotenoids. Approximately 50 exist in nature and the most common provitamin A forms are $\alpha$-carotene, $\beta$-carotene, and $\beta$-cryptoxanthin. All-trans- $\beta$-carotene is a symmetric molecule containing two $\beta$-ionone cyclic ends (Figure 1) and therefore can form two complete VA molecules [2], which is why it is sometimes considered a preferable carotenoid source of VA. Other isomeric forms of $\beta$-carotene can also be converted to VA within the body at a lower rate $[3,4]$. The basis for the theoretical ratios of provitamin $\mathrm{A}$ to $\mathrm{VA}$ is the unique structure of each of the provitamin A carotenoids; however, actual in vivo ratios are dependent upon many factors [3]. The xanthophyll carotenoids, zeaxanthin and lutein, have antioxidant properties due to the polyene structure (Figure 1), but have no VA value because of the hydroxyl groups on both of their rings [5]. However, they have been implicated in the prevention of macular degeneration because they are concentrated in the macula of the eye [6].

Herbal medicine has been used as a curative and preventative measure against various illnesses for centuries in many cultures [7]. Preparations commonly used are herbal water infusions and alcoholic tinctures. Herbal water infusions are gaining popularity because they are beneficial to health due to their high antioxidant content [8]. Infusions are made by adding boiling water to dried herb, sealing the container, and allowing the mixture to steep for a designated amount of time. Various water-soluble compounds leach from the herbs into the water portion of the mixture, also known as the menstruum, which is then consumed [8]. It is unknown to what extent fat-soluble compounds, such as carotenoids, leach into the menstruum.

Herbal alcoholic tinctures are another preparation in which a combination of raw-dried herb and ethanol is used. The liquid menstruum from a tincture is administered in small quantities each day, depending on the age and health of the individual. A study found that when a $50 \%$ alcoholic tincture of Echinacea spp. was compared with a cold and hot water infusion, the tincture showed the greatest immune stimulation when administered to individuals [9].

Calendula flower, and Catnip, Dandelion, Stinging Nettle, and Violet leaves are herbs that are regularly used in herbal medicine but little research has been done to determine the specific carotenoid profile or concentrations. Past research demonstrated that Calendula flower carotenoid content was lower in the tincture than in the dried herb [10]. Carotenoid analysis of Dandelion isolated all-trans-lutein epoxide as the major carotenoid [11]. One study that analyzed phenolic and antioxidant capacities of Catnip found high levels of nepetalactones, which work as a mild sedative, but low levels of antioxidants [12]. High pressure liquid chromatography (HPLC) analysis of Stinging Nettle leaf identified nine carotenoids including lutein, $\beta$-carotene, and their isomers [13]. Stinging Nettle's putative health benefits were recently reviewed [14]. Phenolic compounds, flavonoids, caffeic acid, and salicylic acid were analyzed in a $10 \%$ alcoholic tincture of Violet; high salicylic acid concentrations contributed to antioxidant potency [15]. The current study compares the carotenoid content of these five herbs in the following forms: rawdried herb, water infusion menstruum and marc (spent herb), and alcoholic tincture menstruum and marc. Analyzing raw-dried herbs and various preparative forms adds to the knowledge of how different processing methods impact carotenoid content.

*Corresponding author: Sherry A Tanumihardjo, Department of Nutritional Sciences, 1415 Linden Drive, Madison, WI 53706-1571, USA, Tel: 608-265-0792; Fax: 608-262-5860; E-mail: sherry@nutrisci.wisc.edu

Received October 22, 2014; Accepted November 14, 2014; Published January 03, 2015

Citation: Olsen K, Arscott S, Tanumihardjo SA (2015) Carotenoid Profiles of Dried Herbs, Water Infusions and Alcoholic Tinctures of Calendula Flower and Catnip, Dandelion, Stinging Nettle, and Violet Leaves. Nat Prod Chem Res 3: 160 doi:10.4172/2329-6836.1000160

Copyright: (c) 2015 Olsen K. This is an open-access article distributed under the terms of the Creative Commons Attribution License, which permits unrestricted use, distribution, and reproduction in any medium, provided the original author and source are credited. 
<smiles>CC1=C(/C=C/C=C/C(C)=C/C=C/C=C(C)/C=C/C=C(C)/C=C/C2=C(C)CCCC2(C)C)C(C)(C)CCC1</smiles><smiles>CC1=C(/C=C/C(C)=C/C=C/C(C)=C/C=C/C=C(C)/C=C/C=C(C)/C=C/C2=C(C)CC(O)CC2(C)C)C(C)(C)CC(O)C1</smiles><smiles>CC1=CC(O)CC(C)(C)C1/C=C/C(C)=C/C=C/C(C)=C/C=C/C=C(C)/C=C/C=C(C)/C=C/C1=C(C)CC(O)CC1(C)C</smiles>

\section{lutein}

Figure 1: All-trans- $\beta$-carotene is a symmetric molecule containing two $\beta$-ionone cyclic ends, which allows it to form two vitamin A (VA) molecules. Zeaxanthin and lutein both have hydroxyl groups on their cyclic ends and are therefore not VA precursors but are important to eye health.

\section{Materials and Methods}

\section{Samples and preparations}

Commercially available (CM) herb samples of leaves of Catnip (Nepeta cataria), Dandelion (Taraxacum officinale F. Weber ex Wiggers), Stinging Nettle (Urtica dioica L.) and Violet (Viola odorata), and Calendula flowers (Calendula officinalis L.), were purchased from Community Pharmacy (Madison, WI). Homegrown (HG) Violet leaf and Calendula flowers were provided by Wildwood Institute (Madison, WI). Upon visual analysis, the HG Violet leaf was dark green while the $\mathrm{CM}$ variety was brown. The CM Calendula flower was bright yellow, had larger flowers, and had a much higher ratio of petal to non-petal flower parts than the HG variety. The whole raw-dried herbs were stored in plastic bags at $-30^{\circ} \mathrm{C}$ until use.

The HG Violet leaves and Calendula flowers were compared with their CM variety counterparts only in the raw-dried form. All of the $\mathrm{CM}$ varieties were analyzed in the raw-dried form, water infusion, and alcoholic tincture preparations. CM raw-dried herbs were ground with mortar and pestle before tincture and infusion preparations and lab analyses. The water infusions were prepared in half-pint mason jars$192 \mathrm{ml}$ distilled boiling water was added to each jar containing $6.95 \mathrm{~g}$ herb. The mixture was left to infuse for 8 hours, kept in a dark area at room temperature, and then decanted by straining the herb from the menstruum, the liquid portion of the infusion. The marc $(0.1 \mathrm{~g})$, which is the spent herb and the menstruum $(2.0 \mathrm{ml})$ were placed in glass test tubes and analyzed immediately. The remaining infusion menstruum was stored at $-30^{\circ} \mathrm{C}$ in an amber vial to minimize light exposure and the remaining marc was stored at $-80^{\circ} \mathrm{C}$.

The alcoholic tinctures were prepared in a 1:5 herb to menstruum ratio in half-pint mason jars. Each herb (14 g) was weighed and then combined with $70 \mathrm{~g} 50 \%$ ethanol in a paraffin wax sealed jar and shaken once a day for 14 days. After 14 days, the tinctures were decanted by straining the herb from the menstruum. The menstruum $(2.0 \mathrm{ml} /$ tube $)$ was analyzed immediately. The marc was stored at $-80^{\circ} \mathrm{C}$ in plastic test tubes and the remaining menstruum was stored in amber vials at $-30^{\circ} \mathrm{C}$.

\section{Saponification}

The saponification methods used for the raw-dried herb were adapted from Kurilich and Juvik [16]. Each sample was analyzed in triplicate. Ethanol $(6 \mathrm{ml})$ with $0.1 \%$ butylated hydroxytoluene as antioxidant was added to $0.1 \mathrm{~g}$ raw-dried herb in a $50 \mathrm{ml}$ test tube. The sample was mixed with a vortex for $20 \mathrm{sec}$ and placed in a $65^{\circ} \mathrm{C}$ water bath for $5 \mathrm{~min}$, with the exception of Calendula flower, which was saponified at $85^{\circ} \mathrm{C}$ to maximize carotenoid extraction. The lid was kept loose during the saponification procedure to allow hot air to vent with minimal loss of ethanol. After $500 \mu \mathrm{l}$ potassium hydroxide:water (80:20, w:v) was added, the sample was mixed with a vortex for 20 sec and placed back in the water bath for $5 \mathrm{~min}$. The test tubes were removed, mixed with a vortex for $20 \mathrm{sec}$, and placed in the hot water bath for a final $5 \mathrm{~min}$. Immediately after, the test tubes were placed in ice, $4 \mathrm{ml}$ cold, distilled water was added, mixed with a vortex for 20 sec, and placed back on ice. $\beta$-apo-8'-carotenal $(400 \mu \mathrm{l})$, the internal standard used to account for mechanical loses, was added to the second and third test tubes of each triplicate set and mixed with a vortex for 20 sec. Internal standard was not added to one tube in case of co-elution with a carotenoid of interest.

The saponification methods used for the infusion and tincture marc $(0.1 \mathrm{~g})$ and menstruum $(2.0 \mathrm{ml})$ were the same as the methods described for the raw-dried herb, except samples were saponified at room temperature.

\section{Extraction procedures}

After saponification, $4 \mathrm{ml} \mathrm{HPLC}$-grade mixed hexanes was added to each test tube. The tube was mixed with a vortex for $20 \mathrm{sec}$ and centrifuged for $2 \mathrm{~min}$. Using a glass pipette, the top hexane layer was transferred to a $50 \mathrm{ml}$ test tube. These steps were repeated 2 more times with $3 \mathrm{ml}$ hexanes, except 3 additional extractions were used for rawdried Calendula flower to optimize recovery.

The extract was washed with $4 \mathrm{ml}$ distilled water, intermittently mixed with a vortex 3-4 times ( $1 \mathrm{sec} /$ time), and centrifuged for 2 min. A glass pipette was used to remove the top organic layer to a $25 \mathrm{ml}$ test tube. The water wash was extracted 2 more times with 2 $\mathrm{ml}$ hexanes. The total extract was dried under $\mathrm{N}_{2}$, reconstituted in $500 \mu \mathrm{l}$ 50:50 (v:v) methanol:dichloroethane, mixed with a vortex for $20 \mathrm{sec}$, transferred to an HPLC vial, and $50 \mu \mathrm{l}$ was injected into the HPLC system [17].

\section{High pressure liquid chromatography system}

Three internal standard vials were placed in the sequence for HPLC analysis, two at the start of the run and one after the samples. The HPLC procedure was adapted from Howe and Tanumihardjo [17]. The analytical column was the C30 YMC Carotenoid column (4.6 x $250 \mathrm{~mm}, 3 \mu \mathrm{m}$, Waters Corp., Milford, MA) equipped with a guard column. Solvent A contained 92:8 methanol:water (v:v) with 10 mM ammonium acetate as modifier and Solvent B was $100 \%$ methyltertiary-butyl ether. Linear gradient elution was performed at $1 \mathrm{ml} /$ min: $0-30$ min beginning with $70 \% \mathrm{~A}: 30 \% \mathrm{~B}$ to $40 \% \mathrm{~A}: 60 \% \mathrm{~B}$, with a transition back to $70 \% \mathrm{~A}: 30 \% \mathrm{~B}$ at 32 min for equilibration.

Carotenoids were identified by their retention time in comparison with HPLC-purified standards and their characteristic absorption spectra generated by the HPLC, which was equipped with a photodiode array detector [17]. Each characteristic three-peak spectrum was evaluated and matched with the corresponding carotenoid to determine the overall carotenoid profile (Table 1).

\section{Statistical analysis}

Recovery of the internal standard within the second and third test tubes was determined with the pure internal standard injections. With 

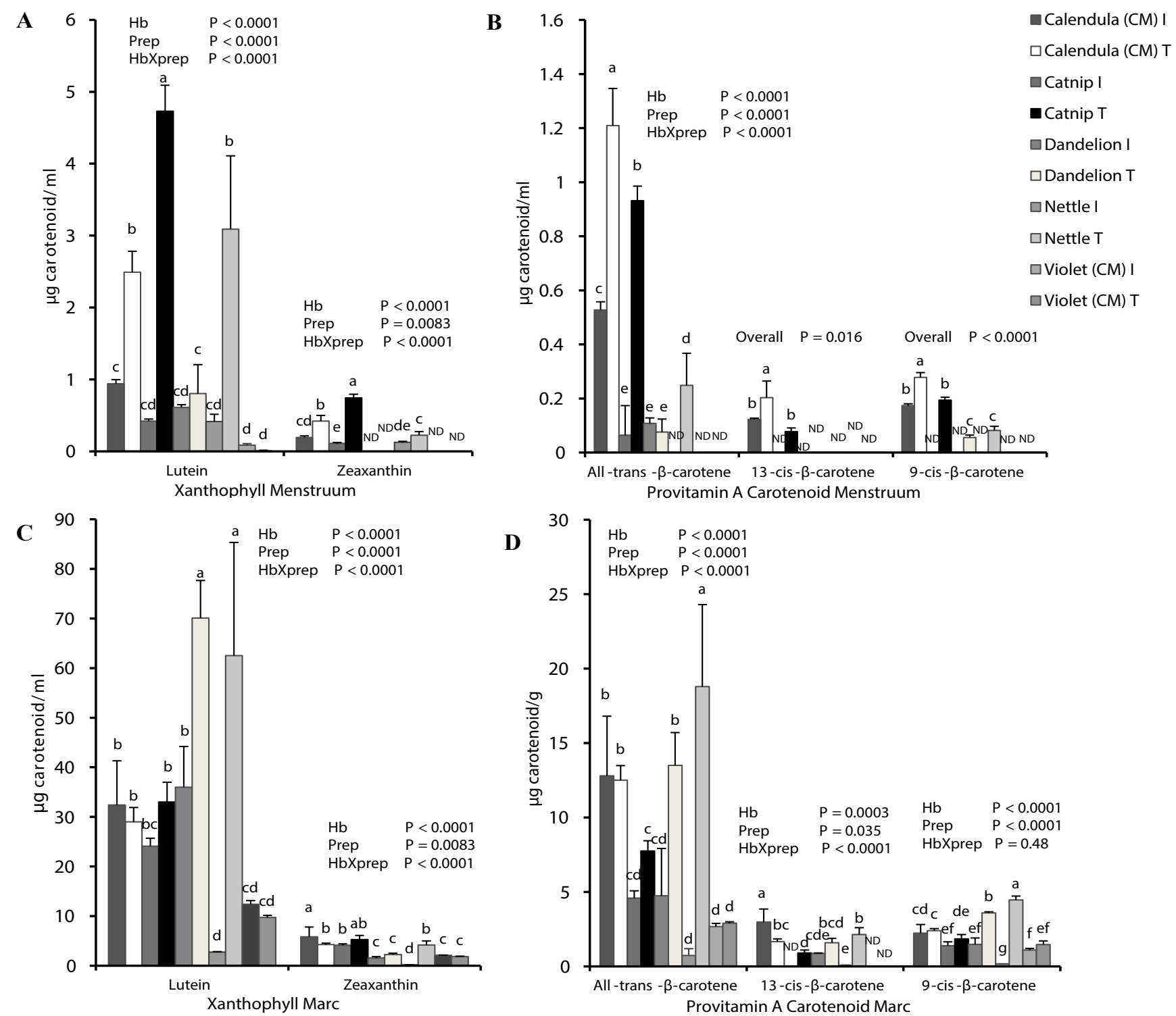

Figure 2: Comparison of herbal preparation carotenoid profiles in the menstrua and the marcs. Based on LSD comparisons, different letters within a grouping (carotenoid category) signify differences and includes the water infusions and alcoholic tinctures $(P<0.05)$. Interactions between herb $(\mathrm{Hb})$ and preparation (prep), infusion (I) or tincture (T), were significant for all carotenoids $(P<0.0001)$, except for 9 -cis- $\beta$-carotene $(P=0.48)$. Note that the scales are not the same on each graph.

the recovery taken into account, all carotenoids in each preparation were expressed as a mean concentration $\pm \mathrm{SD}$. One- and two-way ANOVA were used where appropriate to compare differences among carotenoid concentrations in the herbs and preparations and to evaluate any interactions. LSD was used to determine differences between herb carotenoid values. $P \leq 0.05$ was considered significant. The percent loss due to processing was calculated by taking the individual raw-dried herb carotenoid data and comparing it with the individual carotenoid concentration from each preparation.

\section{Results}

\section{Raw-dried herb}

The total carotenoid composition for the raw-dried herbs is shown in Table 1. Significant differences were found among all analyzed carotenoids by herb type (all $P<0.0001$ ). A two-way
ANOVA was performed and showed no significant effect of herb and source on total carotenoid content. However, an interaction occurred between herb type and source for lutein, zeaxanthin, and 9-cis- $\beta$-carotene (all $P<0.0001$ ). A sub-analysis showed no effect of the HG variety compared with the CM varieties of Calendula flower and Violet leaf for total carotenoid content $(P=0.056)$ or procurement source $(P=0.20)$.

Disregarding the HG varieties, one-way ANOVA revealed differences in the carotenoid content among all $\mathrm{CM}$ varieties $(P$ $<0.0001)$. The total carotenoid content was highest in Calendula flower followed by Dandelion, Stinging Nettle, Catnip, and Violet leaves. Lutein concentrations differed $(P<0.0001)$; Calendula's lutein concentration was higher than Dandelion's, followed by Stinging Nettle's, and then Catnip's and Violet's, which did not differ from each other $(P<0.05)$. Calendula's zeaxanthin concentration was 


\begin{tabular}{|c|c|c|c|c|c|c|c|c|}
\hline Carotenoid & Calendula (CM) & Calendula (HG) & Catnip & Dandelion $\mu \mathrm{g} / \mathrm{g}$ & Stinging Nettle & Violet (CM) & Violet (HG) & $P$ values \\
\hline Lutein & $215 \pm 6.89^{a}$ & $8.23 \pm 7.29^{e}$ & $42.0 \pm 3.42^{\mathrm{d}}$ & $119 \pm 22.7^{b}$ & $79.3 \pm 3.09^{c}$ & $25.3 \pm 3.13^{\text {de }}$ & $238 \pm 41.1^{a}$ & $<0.0001$ \\
\hline Zeaxanthin & $35.7 \pm 2.20^{a}$ & $1.54 \pm 0.34^{e}$ & $7.30 \pm 0.63^{c}$ & $3.06 \pm 0.31^{\mathrm{de}}$ & $4.42 \pm 0.16^{d}$ & $3.07 \pm 0.10^{\text {de }}$ & $11.9 \pm 2.11^{b}$ & $<0.0001$ \\
\hline All-trans- $\beta$-carotene & $36.7 \pm 2.18^{b}$ & $1.28 \pm 0.22^{\mathrm{d}}$ & $8.32 \pm 1.06^{\mathrm{cd}}$ & $19.8 \pm 2.66^{c}$ & $19.0 \pm 0.71^{c}$ & $\mathrm{ND}^{*}$ & $100 \pm 17.2^{\mathrm{a}}$ & $<0.0001$ \\
\hline 13 -cis- $\beta$-carotene & $6.89 \pm 0.26^{a}$ & $1.28 \pm 0.25^{c}$ & $1.64 \pm 0.32^{c}$ & $1.82 \pm 0.88^{c}$ & $2.71 \pm 0.11^{b}$ & ND & $3.36 \pm 0.55^{b}$ & $<0.0001$ \\
\hline 9-cis- $\beta$-carotene & $6.60 \pm 1.21^{b}$ & $1.04 \pm 0.17^{e}$ & $2.06 \pm 0.20^{\mathrm{de}}$ & $4.24 \pm 0.72^{c}$ & $3.86 \pm 0.24^{\mathrm{cd}}$ & $1.45 \pm 0.23^{e}$ & $15.7 \pm 2.60^{\mathrm{a}}$ & $<0.0001$ \\
\hline Total carotenoids & $301 \pm 87.9^{b}$ & $13.4 \pm 3.11^{\mathrm{e}}$ & $61.3 \pm 16.9^{d}$ & $148 \pm 50.7^{c}$ & $109 \pm 32.8^{c}$ & $29.8 \pm 10.9^{d e}$ & $370 \pm 100^{a}$ & $<0.0001$ \\
\hline
\end{tabular}

Means without a common superscript letter in a row differ, $P<0.05$.

$P$ values are indicated for overall differences within each carotenoid.

Table 1: Carotenoid profile (Mean \pm SD) of the raw-dried herbs

higher than Catnip's, followed by Dandelion's, Stinging Nettle's, and Violet's, which did not differ from each other $(P<0.05)$. Calendula's all-trans- $\beta$-carotene concentration was higher than Dandelion's and Stinging Nettle's, which did not differ from each other, followed by Catnip's $(P<0.05)$. Calendula's 13 -cis- $\beta$-carotene concentration was higher than Stinging Nettle's and Dandelion's, which did not differ from each other, and Catnip's, which did not differ from Dandelion's $(P<0.05)$. Calendula's 9-cis- $\beta$-carotene concentration was higher than Dandelion's and Stinging Nettle's, which did not differ from each other, followed by Catnip's and Violet's, which did not differ from each other $(P<0.05)$

\section{Carotenoids not transferred}

Due to the steeping process, 24.9 to $97.2 \%$ of carotenoids were lost during infusion and 0 to $86.9 \%$ of carotenoids were lost during tincture preparation (Table 2). The infusion steeping process uses boiling water and the alcoholic tincture steeps for a longer period of time. Thus, $\%$ carotenoids lost was often substantial and highly variable due to destruction by heat or over time.

\section{Infusion and tincture menstrua}

Total carotenoid concentrations differed among all herb menstrua (overall $P<0.0001$; Table 3). Similar patterns of difference between herbs were found for lutein, zeaxanthin and all-trans- $\beta$-carotene concentrations in the infusion and tincture menstrua $(P<0.05$; Figure $2 \mathrm{AB})$. Not all groups had detectable 9-cis- and 13-cis- $\beta$-carotene concentrations in the menstrua (Figure $2 \mathrm{~B}$ ). The tincture menstrua had higher carotenoid concentrations than the infusion menstrua $(P \leq 0.0083)$. An herb type by preparation interaction existed for lutein, zeaxanthin, and all-trans- $\beta$-carotene concentrations $(P<0.0001$; Figure $2 \mathrm{AB})$.

Between the infusion menstrua, lutein concentration was higher in Calendula than Violet, while Catnip, Dandelion, and Stinging Nettle did not differ from either $(P<0.05)$. Zeaxanthin concentration was higher in Calendula than Catnip, and Stinging Nettle did not differ from either $(P<0.05)$. All-trans-, 13-cis-, and 9-cis- $\beta$-carotene concentrations were highest in Calendula (Figure 2B). All-trans$\beta$-carotene concentration was higher in Calendula than Catnip and Dandelion $(P<0.05)$. Between the tincture menstrua, lutein concentration was higher in Catnip than in Calendula and Stinging Nettle, which did not differ from each other, followed by Dandelion and Violet $(P<0.05)$. Zeaxanthin concentration was higher in Catnip than Calendula and Stinging Nettle $(P<0.05)$. All-trans- $\beta$-carotene, 13-cis- $\beta$-carotene, and 9-cis- $\beta$-carotene concentrations were highest in Calendula tincture menstruum compared with Catnip, Dandelion, and Stinging Nettle (Figure 2B; $P<0.05$ ). Provitamin A carotenoids were not detectable in Violet's menstruum.

\section{Infusion and tincture marcs}

All herb marc carotenoid concentrations differed (overall $P=$
0.0003; Table 3). The highest carotenoids remained in Calendula's and Dandelion's infusion marcs, followed by Catnip's, Violet's, and Stinging Nettle's $(P<0.05)$. Total carotenoids were highest in the Dandelion's and Stinging Nettle's tincture marcs followed by Calendula's and Catnip's, which did not differ from each other $(P<0.05)$.

Two-way ANOVA revealed a significant effect of herb type on individual carotenoids left in the marcs (Figure 2CD). Herb type by preparation interactions existed for the carotenoids $(P<0.0001)$, with the exception of 9 -cis- $\beta$-carotene. Lutein concentrations differed among preparations $(P<0.0001)$ and were generally higher in the tincture marcs when compared with the infusion marcs $(P<0.0001$; Figure 2C). Herb type and preparation significantly impacted lutein and zeaxanthin concentrations (all $P \leq 0.0083$ ). In an overall comparison, zeaxanthin concentration was highest in the Calendula infusion marc $(P<0.0001)$. All-trans-, $13-c i s$, and 9-cis- $\beta$-carotene concentrations in the marcs differed among herb type $(P \leq 0.0003)$, and tincture marc had the highest concentrations $(P \leq 0.035)$.

Between the infusion marcs, lutein concentration was higher in Calendula, Catnip, and Dandelion, which did not differ, followed by Violet and Stinging Nettle, which did not differ $(P<0.05)$. Zeaxanthin concentration was higher in Calendula than Catnip, followed by Violet and Dandelion, which did not differ, and Stinging Nettle's was the lowest $(P<0.05)$. All-trans- $\beta$-carotene concentration was higher in Calendula than Catnip, Dandelion, Stinging Nettle, and Violet, which did not differ $(P<0.05)$. 13-cis- $\beta$-carotene concentration was higher in Calendula than Dandelion and Stinging Nettle, which did not differ $(P<0.05) .9-c i s-\beta$-carotene concentration was higher in Calendula than Catnip, Dandelion, and Violet, which did not differ, and Stinging Nettle had the lowest concentration $(P<0.05)$.

Between tincture marcs, Dandelion's and Stinging Nettle's lutein concentrations were higher than Calendula's and Catnip's, which did not differ from each other, and Violet's was the lowest but did not differ from Catnip's $(P<0.05)$. Calendula's, Catnip's, and Stinging Nettle's zeaxanthin concentrations were highest, followed by Dandelion's and Violet's, which did not differ from each other $(P<0.05)$. All-trans- $\beta$ carotene concentration was higher in Stinging Nettle than Dandelion and Calendula, which did not differ, followed by Catnip, and Violet was the lowest $(P<0.05)$. 13-cis- $\beta$-carotene concentration was highest in Stinging Nettle, Calendula, and Dandelion, which did not differ, and Dandelion's concentration did not differ from Catnip's $(P<0.05)$. 9-cis--carotene concentration was highest in Stinging Nettle followed by Dandelion, than Calendula, and Catnip and Violet concentrations were the lowest and did not differ from each other $(P<0.05)$.

\section{Discussion}

Homegrown Violet had the highest mean concentration of total carotenoids within the raw-dried herb samples and therefore might be 
Citation: Olsen K, Arscott S, Tanumihardjo SA (2015) Carotenoid Profiles of Dried Herbs, Water Infusions and Alcoholic Tinctures of Calendula Flower and Catnip, Dandelion, Stinging Nettle, and Violet Leaves. Nat Prod Chem Res 3: 160. doi:10.4172/2329-6836.1000160

Page 5 of 6

\begin{tabular}{|c|c|c|c|c|c|c|}
\hline & \multicolumn{3}{|c|}{ Water infusion } & \multicolumn{3}{|c|}{ Alcoholic tincture } \\
\hline & Menstruun & Marc & Total compared to raw-dried herb & Menstruum & Marc & Total compared to raw-dried herb \\
\hline & \multicolumn{2}{|c|}{ Carotenoid retained } & Lost & \multicolumn{2}{|c|}{ Carotenoid retained } & Lost \\
\hline \multicolumn{7}{|c|}{$\%$} \\
\hline \multicolumn{7}{|l|}{ Calendula flower } \\
\hline Lutein & 0.44 & 15.0 & 84.5 & 1.16 & 13.5 & 85.4 \\
\hline Zeaxanthin & 0.54 & 16.4 & 83.1 & 1.18 & 12.0 & 86.9 \\
\hline All-trans- $\beta$-carotene & 1.44 & 34.8 & 63.8 & 3.29 & 34.0 & 62.7 \\
\hline 13-cis- $\beta$-carotene & 1.78 & 32.5 & 65.7 & 2.95 & 24.1 & 72.9 \\
\hline 9-cis- $\beta$-carotene & 2.63 & 33.8 & 63.5 & 4.21 & 36.3 & 59.5 \\
\hline \multicolumn{7}{|l|}{ Catnip } \\
\hline Lutein & 1.00 & 57.3 & 41.7 & 11.3 & 78.6 & 10.2 \\
\hline Zeaxanthin & 1.50 & 56.5 & 42.0 & 10.2 & 72.1 & 17.7 \\
\hline All-trans- $\beta$-carotene & 0.76 & 55.2 & 44.1 & 11.2 & 93.1 & 0 \\
\hline 13-cis- $\beta$-carotene & ND & ND & & 4.73 & 54.1 & 41.1 \\
\hline 9-cis- $\beta$-carotene & ND & 67.5 & 36.5 & 9.39 & 89.7 & 0.87 \\
\hline \multicolumn{7}{|l|}{ Dandelion } \\
\hline Lutein & 0.51 & 30.2 & 69.4 & ND & 58.7 & 40.6 \\
\hline Zeaxanthin & ND & 51.1 & 49.0 & ND & 72.9 & 26.4 \\
\hline All-trans- $\beta$-carotene & 0.55 & 23.9 & 75.5 & 0.38 & 68.3 & 31.3 \\
\hline 13-cis- $\beta$-carotene & ND & 47.8 & 68.2 & ND & 87.1 & 13.1 \\
\hline 9-cis- $\beta$-carotene & ND & 34.6 & 65.4 & 1.32 & 84.6 & 14.1 \\
\hline \multicolumn{7}{|l|}{ Stinging Nettle } \\
\hline Lutein & 0.52 & 3.49 & 96.0 & 7.26 & 78.8 & 17.3 \\
\hline Zeaxanthin & 2.82 & 4.30 & 92.9 & 5.05 & 94.7 & 0.30 \\
\hline All-trans- $\beta$-carotene & ND & 3.83 & 96.2 & ND & 99.0 & 1.10 \\
\hline 13-cis- $\beta$-carotene & ND & 2.76 & 97.2 & ND & 78.7 & 21.3 \\
\hline 9-cis- $\beta$-carotene & ND & 4.02 & 96.0 & 2.12 & 100 & 0 \\
\hline \multicolumn{7}{|l|}{ Violet } \\
\hline Lutein & 0.33 & 48.9 & 50.7 & ND & 38.4 & 61.1 \\
\hline Zeaxanthin & ND & 68.7 & 31.3 & ND & 60.2 & 39.8 \\
\hline All-trans- $\beta$-carotene & ND & ND & & ND & ND & \\
\hline 13-cis- $\beta$-carotene & ND & ND & & ND & ND & \\
\hline 9-cis- $\beta$-carotene & ND & 75.3 & 24.7 & ND & 100 & 0 \\
\hline \multicolumn{7}{|c|}{ ND, not detected } \\
\hline
\end{tabular}

Table 2: Percent carotenoids retained in herbal preparations and percent loss due to processing.

\begin{tabular}{|c|c|c|c|c|c|c|}
\hline Preparation & Calendula (CM) & Catnip & Dandelion & Stinging Nettle & Violet (CM) & $P$ values \\
\hline Dried herb $(\mu \mathrm{g} / \mathrm{g})$ & $301 \pm 87.9^{a}$ & $61.3 \pm 16.9^{d}$ & $148 \pm 50.7^{b}$ & $109 \pm 32.8^{c}$ & $29.8 \pm 10.9^{\mathrm{e}}$ & $<0.0001$ \\
\hline \multicolumn{7}{|l|}{ Infusion } \\
\hline Menstruum $(\mu \mathrm{g} / \mathrm{ml})$ & $1.96 \pm 0.35^{a}$ & $0.62 \pm 0.17^{c}$ & $0.718 \pm 0.265^{b}$ & $0.538 \pm 0.179^{c}$ & $0.083 \pm 0.037^{d}$ & $<0.0001$ \\
\hline Marc $(\mu \mathrm{g} / \mathrm{g})$ & $56.2 \pm 12.5^{\mathrm{a}}$ & $34.2 \pm 9.8^{\mathrm{bc}}$ & $44.3 \pm 15.2^{\mathrm{ab}}$ & $3.92 \pm 1.14^{\mathrm{d}}$ & $18.3 \pm 4.99^{\text {cd }}$ & 0.0003 \\
\hline \multicolumn{7}{|l|}{ Tincture } \\
\hline Menstruum $(\mu \mathrm{g} / \mathrm{ml})$ & $4.60 \pm 0.58^{b}$ & $6.67 \pm 0.48^{\mathrm{a}}$ & $0.955 \pm 0.49^{c}$ & $3.65 \pm 1.21^{b}$ & $0.130 \pm 0.008^{c}$ & $<0.0001$ \\
\hline $\operatorname{Marc}(\mu \mathrm{g} / \mathrm{g})$ & $49.8 \pm 11.5^{b}$ & $48.8 \pm 13.3^{b}$ & $91.1 \pm 29.4^{\mathrm{a}}$ & $92.1 \pm 25.5^{\mathrm{a}}$ & $16.3 \pm 3.73^{c}$ & 0.0003 \\
\hline
\end{tabular}

Means without a common superscript letter in a row differ, $P<0.05$.

Table 3: Total carotenoids (Mean \pm SD) in all of the commercial samples analyzed.

a better source of carotenoids than CM when processed. HG variety menstrua were not prepared because of non-availability to the general public. HG Violet, which was supplied as dark green, whole leaves, had more total carotenoids than the CM Violet, which was supplied in a dried, crushed format and was duller brown-green. CM Calendula flower had the second highest amount of total carotenoids. This CM Calendula flower, which was more vibrant yellow with a higher ratio of petals to non-petal flower parts, had more total carotenoids than the HG sample that was not as bright yellow and had fewer petals to non-petals. Color is an important aspect for consumers in selecting the freshest herbs, as reduced color vibrancy likely reflects loss of carotenoids and may also reflect degradation of other desirable phytochemicals [18].

The differences among the herbs for the five carotenoids were all significant indicating a disparity; therefore, certain herbs may be preferential to others for individual carotenoids. For example, raw-dried HG Violet showed the highest mean concentration for $\beta$-carotene and therefore had the highest VA activity possible among the herbs, whereas CM Violet leaves had non-detectable levels of all-trans- $\beta$-carotene. Large variation in carotenoids likely exists among different suppliers of rawdried herbs. There was also a difference in carotenoid profiles between the infusion and tincture preparations within the same herb.

The mean concentrations of all the carotenoids for the marcs of both the herbal infusion and tincture were less than the raw-dried herb due to leaching into the menstrua and loss during processing. The tincture marcs with the highest mean concentrations of total carotenoids were Dandelion and Stinging Nettle. Whereas for the infusion 
marc, Dandelion and Calendula had the highest concentrations of total carotenoids, and Stinging Nettle had the lowest mean total carotenoid concentration. This may be due to differences in herb composition or matrix, which influence the ability to extract carotenoids. Differences between the polarity of water and alcohol may have also played a role in carotenoid extraction. The total mean carotenoid concentration changed with each preparation, and the marcs contained more total carotenoids than the menstrua for both the tinctures and infusions. It is interesting to note that there was discrepancy among the different herbs in \% carotenoid lost during processing. Future studies should more closely assess these differences, which may include analysis of chlorophyll content, alcoholic compounds, such as glycosides and sterols, other vitamins, and minerals.

The tincture and infusion menstrua had the lowest mean concentration of total carotenoids $(<2 \mu \mathrm{g}$ total carotenoid/ml $)$, compared with the raw-dried herbs and marcs. The tincture menstrua had higher mean concentrations of total carotenoids across all herbs analyzed than the water infusions. This is likely due to the use of alcohol as a solvent and the longer steeping time used in preparing a tincture, 14 days vs. 8 hours for an infusion. Considering that orange carrot has $\sim 90 \mu \mathrm{g} \beta$-carotene/g and spinach has $\sim 120 \mu$ g lutein plus zeaxanthin/g [19], herbal water and alcoholic preparations are likely not a significant dietary source of carotenoids. While it is commonly known that carotenoids are fatsoluble compounds that are not readily released into polar solvents, these findings support this fact and will serve as a useful reference for consumers and holistic health practitioners.

This study supports past research that found lower concentrations of carotenoids in Calendula tincture than in the raw-dried herb [10]. Lutein was also identified as the major carotenoid in Calendula and Dandelion [11]. Moreover, this study gives further evidence that the raw-dried herb has the highest mean carotenoid concentrations relative to the infusions and tinctures. Further research should be done to determine which herbal forms have the most bioavailable carotenoids to the human body to show which would be best to derive nutritional benefits.

\section{Conclusion}

The concentrations of all-trans- $\beta$-carotene, 9 - and 13 -cis- $\beta$-carotene, zeaxanthin and lutein varied throughout the different preparations. These variances suggest differing antioxidant and VA potentials. The raw-dried herb showed the highest concentrations of total carotenoids with the darker, green leaves of HG Violet having higher concentrations than the duller CM Violet. Of the preparations used in herbal medicine, the tincture and infusion menstrua had lower total carotenoid content than the raw-dried herbs and marcs. Additionally, the concentrations of the individual carotenoids varied between the two preparations and across the herbs and did not always reflect the carotenoid profile of the raw-dried herb. Determining carotenoid composition is important to identify which preparation is best suited for specific medicinal purposes. When looking specifically at VA activity, the highest $\beta$-carotene concentration was in Calendula's tincture menstruum preparation.

\section{Acknowledgement}

The authors would like to thank Kara Bresnahan for assistance in the statistical analysis of the data, Kathleen Wildwood from Wildwood Institute who inspired us to evaluate VA claims of herbal preparations, and Devika Suri for editorial assistance. This work was supported by an endowment to SAT entitled
"Friday Chair for Vegetable Processing Research." This was an undergraduate research project for KO and was partially supported by the Cargill-Benevenga Scholarship for undergraduate researchers.

\section{References}

1. Darlow BA, Graham PJ (2011) Vitamin A supplementation to prevent mortality and short- and long-term morbidity in very low birth weight infants. Cochrane Database Syst Rev CD000501.

2. Tapiero H, Townsend DM, Tew KD (2004) The role of carotenoids in the prevention of human pathologies. Biomedicine and Pharmacotherapy 58: 100-110.

3. Tanumihardjo SA, Palacios N, Pixley KV (2010) Provitamin A carotenoid bioavailability: What really matters? International Journal of Vitamin and Nutrition Research 80: 336-350.

4. Bresnahan KA, Davis CR, Tanumihardjo SA (2014) Relative vitamin A values of 9-cis- and 13-cis-b-carotene do not differ when fed at physiological levels during vitamin A depletion in Mongolian gerbils (Meriones unguiculatus) British Journal of Nutrition 112: 162-169.

5. Stahl W, Sies H (2003) Antioxidant activity of carotenoids. Molecular Aspects of Medicine 24: 345-351.

6. Vishwanathan R, Johnson EJ (2013) Lutein and zeaxanthin and eye disease In Carotenoids and Human Health. Edited by Tanumihardjo SA. New York: Springer Science and Business Media.

7. Ferreira Jr WS, Cruz MP, dos Santos LL, Medeiros MFT (2012) Use and importance of quina (Cinchona spp.) and ipeca (Carapichea ipecacuanha (Brot.) L. Andersson): Plants for medicinal use from the 16 th century to the present. Journal of Herbal Medicine 2: 103-112.

8. Atoui A, Mansouri A, Boskou G, Kefalas P (2005) Tea and herbal infusions: Their antioxidant activity and phenolic profile. Food Chemistry 89: 27-36.

9. Senchina DS, McCann DA, Asp JM, Johnson JA, Cunnick JE, et al. (2005) Changes in immunomodulatory properties of Echinacea spp. root infusions and tinctures stored at 4 degrees $C$ for four days. Clinica Chimia Acta 355: 67-82.

10. Bako E, Deli J, Toth G (2002) HPLC study on the carotenoid composition of Calendula products. Journal of Biochemical and Biophysical Methods 53: 241-250.

11. Melendez-Martinez AJ, Britton G, Vicario IM, Heredia FJ (2006) HPLC analysis of geometrical isomers of lutein epoxide isolated from Dandelion (Taraxacum officinale F. Weber ex Wiggers). Phytochemistry 67: 771-777.

12. Adiguzel A, Ozer H, Sokmen M, Gulluce M, Sokmen A, et al. (2009) Antimicrobial and antioxidant activity of the essential oil and methanol extract of Nepeta cataria. Polish Journal of Microbiology 58: 69-76.

13. Guil-Guerrero JL, Rebolloso-Fuentes MM, Torija Isasa ME (2003) Fatty acids and carotenoids from Stinging Nettle (Urtica Dioica L.). Journal of Food Composition and Analysis 16: 111-119.

14. Upton R (2013) Stinging Nettles leaf (Urtica dioica L.): Extraordinary vegetable medicine. Journal of Herbal Medicine 3: 9-38.

15. Ebrahimzadeh MA, Nabavi SM, Nabavi SF, Bahramian F, Bekhradnia AR (2010) Antioxidant and free radical scavenging activity of H. Officinalis L. Var. Angustifolius V. Odorata, B. Hyrcana and C. Speciosum. Pak J Pharm Sci 23: 29-34.

16. Kurilich AC, Juvik JA (1999) Quantification of carotenoid and tocopherol antioxidants in Zea mays. Journal of Agricultural and Food Chemistry 47: 1948-1955.

17. Howe JA, Tanumihardjo SA (2006) Evaluation of analytical methods for carotenoid extraction from biofortified maize (Zea mays sp.). J Agric Food Chem 54: 7992-7997.

18. Mahanom H, Azizah A, Dzulkifly M (1999) Effect of different drying methods on concentrations of several phytochemicals in herbal preparation of 8 medicinal plants leaves. Malaysian Journal of Nutrition 5: 47-54.

19. Arscott SA (2013) Food sources of carotenoids. In Carotenoids and Human Health. Edited by Tanumihardjo SA. New York: Springer Science and Business Media. 